\title{
Synthesis of Kapakahines B and F
}

Key words

\section{kapakahines}

indoles

annulation

macrocycles

$\alpha$-carbolines

palladium
Significance: Kapakahines are metabolites of the sponge Cribrochalina olemda. Kapakahine B has modest antileukemic activity but kapakahine $F$ is inactive. A salient feature of this short and efficient synthesis is the in situ kinetic trapping of the $\alpha$ carboline $\mathbf{G}$ which is in dynamic equilibrium with the pyrroloindoline $\mathbf{F}$.
Comment: Cyclization of the amino acid $\mathbf{F}$ (participating atoms highlighted in red) gives a macrocycle (not shown) in 6\% yield together with the desired $\alpha$-carboline $\mathbf{G}$. All the steps leading up to $\mathbf{H}$ were performed on a gram scale.

SYNFACTS Contributors: Philip Kocienski 